\title{
Sleep apnoea and visceral adiposity in middle-aged male and female subjects
}

\author{
Ilia Kritikou*, Maria Basta*, Rafel Tappouni”, Slobodanha Pejovic*, \\ Julio Fernandez-Mendoza*, Racha Nazir*, Michelle L. Shaffer", Duanping Liao", \\ Edward O. Bixler*, George P. Chrousos ${ }^{+}$and Alexandros N. Vgontzas*
}

ABSTRACT: In obese male subjects, visceral adiposity has been associated with obstructive sleep apnoea (OSA), while studies in overweight males and females are limited. Our goal was to examine the association between OSA and visceral fat in a relatively nonobese population and assess the effects of 2 months placebo-controlled continuous positive airway pressure (CPAP) use on abdominal fat.

81 subjects, 22 middle-aged males and 20 post-menopausal females with OSA, and 19 male and 20 female controls were studied in the sleep laboratory for four nights. Abdominal (visceral (VAT) and subcutaneous (SAT) adipose tissue) and liver fat were assessed with computed tomography. OSA patients were re-assessed post-CPAP and post sham-CPAP.

Apnoeic males had significantly higher VAT than controls, while apnoeic females had higher SAT than controls. In both sexes, OSA was associated with increased liver fat. In males, apnoea was associated with VAT whereas in females it was associated with subcutaneous, visceral and total fat. CPAP did not affect abdominal and liver fat.

In overweight males, visceral adiposity is associated with OSA whereas in females it is associated with global adiposity. In overweight males, our therapeutic goal should be the reduction of visceral adiposity and its metabolic correlates, whereas, in females, weight loss may be sufficient. Shortterm CPAP treatment does not affect general, abdominal or intra-hepatic adiposity.

KEYWORDS: Abdominal fat, continuous positive airway pressure, post-menopausal, sleepdisordered breathing, visceral adiposity

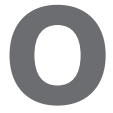
bstructive sleep apnoea (OSA), a common sleep disorder, is most frequent in obese middle-aged males [1]. However, in general population samples, $50 \%$ of apnoeic males are nonobese, while $2 \%$ of females suffer from sleep apnoea with a four-fold increase in the prevalence of OSA post-menopause [2].

OSA is associated with features typical of the metabolic syndrome, i.e. insulin resistance, smouldering systemic inflammation and excessive visceral adiposity [3]. Studies in obese males [3-5] and one study in severely obese females [5] with OSA have shown that visceral adiposity is significantly higher than body mass index (BMI)-matched controls. However, there is only one study in a nonobese Japanese population, which showed that OSA patients had higher amounts of visceral fat than controls [6], whereas no study has assessed visceral adiposity in nonobese females. This Japanese study included a very small number of nonobese females, which did not allow for an appropriate analysis stratified by sex.

Additionally, the very few studies on continuous positive airway pressure (CPAP) effects on body composition are primarily in obese males, and the results are contradictory [4, 7-9].

In the current study, our primary goal was to examine the role of visceral and intra-hepatic adiposity in OSA in middle-aged males and postmenopausal females and their respective controls. Despite our efforts, our sample of females consisted of slightly obese patients because: 1) in Central Pennsylvania, females are heavier compared with national standards [2]; and 2) females with OSA both in clinical and epidemiological samples are heavier than males [10]. A secondary goal was to assess the effect of placebo-controlled (sham-CPAP) 2-month CPAP treatment on body fat composition.
AFFILIATIONS

*Sleep Research and Treatment Center, Dept of Psychiatry, Pennsylvania State University College of Medicine,

"Dept of Radiology, Gastrointestinal and Abdominal imaging,

Pennsylvania State University College of Medicine, and

"Dept of Public Health Sciences, Pennsylvania State University College of Medicine, Hershey, PA, USA. +First Dept of Pediatrics, National and Kapodistrian University of Athens School of Medicine, Athens, Greece.

CORRESPONDENCE

A.N. Vgontzas

Pennsylvania State University College of Medicine, Dept of Psychiatry H073 500 University Drive

Hershey

PA 17033

USA

E-mail: avx3@psu.edu

Received:

Oct 202011

Accepted after revision: June 072012

First published online: June 272012 


\section{MATERIALS AND METHODS \\ Subjects}

The study was completed by 22 overweight middle-aged males with sleep apnoea, 19 age- and BMI-matched nonapnoeic controls, 20 middle-aged post-menopausal apnoeic females and 20 age-matched controls.

The subjects were recruited from the Sleep Disorders Clinic (Pennsylvania State University College of Medicine, Hershey, PA, USA) and through advertisements from the community. To qualify for the study, apnoeic patients had to have apnoea of sufficient severity to warrant recommendation for treatment [4]. These criteria included an apnoea/hypopnoea index (AHI) of $>10$ events $\cdot h^{-1}$ of sleep for females and $>15$ events $\cdot h^{-1}$ for males. A lower threshold was chosen for females, because females have on average lower indices of respiratory disturbance and tend to manifest symptoms at a lower threshold [11]. Controls had to have AHI $<5$ events $\cdot h^{-1}$, no sleep complaints and percentage sleep time $>75 \%$ during the screening night.

Exclusion criteria included a history of diabetes mellitus type 2 , the use of antiglycaemic agents or fasting glucose blood levels $>126 \mathrm{mg} \cdot \mathrm{dL}^{-1}$ at the time of screening, ongoing infections, rheumatoid arthritis, insomnia, narcolepsy and use of specific medications (psychotropics, steroids, sympathomimetics, or sympatholytics or hormone therapy for females). Alcohol consumption was assessed with two questions: 1) Do you ingest alcohol? 2) If yes, how many drinks per day? Abstainers or light drinkers were defined as those (both males and females) that ingested $<0.25 \mathrm{~g} \cdot \mathrm{day}^{-1}$, and moderate drinkers as males that ingested $<30 \mathrm{~g} \cdot \mathrm{day}^{-1}$ and females that ingested $<20 \mathrm{~g} \cdot$ day $^{-1}$ [12]. None of the female participants ingested more than one "standard drink" per day and none of males more than two [12]. Menopause was defined as selfreported absence of menses for at least 12 months or history of surgically induced menopause (i.e. total hysterectomy with bilateral oophorectomy). The study was approved by the University Institutional Review Board (Pennsylvania State University College of Medicine) and all participants provided a written informed consent.

\section{Procedures}

Sleep laboratory

A thorough medical assessment, including history, physical examination, routine laboratory tests and sleep history was completed for each participant. Anthropometric parameters were obtained and BMI was calculated based on height and bodyweight measured as part of the physical examination. All potential participants were screened in the sleep laboratory for one night for $8 \mathrm{~h}$ using standard polysomnographic procedures [13]. The subjects who met the inclusion criteria were monitored in the sleep laboratory for four consecutive nights (one adaptation and three baseline nights).

The study design included two consecutive 2-month periods of CPAP (Ultra Mirage II Nasal mask; S8 Escape, ResMed, San Diego, CA, USA) or sham-CPAP treatment for OSA patients in a counterbalanced order (i.e. half of them were assigned to the $\mathrm{CPAP} /$ sham-CPAP sequence and the other half to the sham$\mathrm{CPAP} / \mathrm{CPAP}$ sequence). Patients remained blinded to the treatment and they were reassessed at the end of each 2-month period. The sleep records were scored independently of any knowledge of the experimental conditions [13]. Also, the respiratory data were quantified as previously described [3].

\section{Computed tomography scanning}

The objective of these measurements was to assess abdominal visceral, subcutaneous and liver fat compartments. Axial 3-mm sections were taken through the mid-vertebral bodies of L1, L2, L3, L4 and L5 lumbar vertebrae. The specific levels to be scanned were localised from an initial sagittal topogram. The scans were performed using a somatom 16-slice MDCT scanner (Siemens Medical solutions, Malvern, PA, USA) using $120 \mathrm{kV}$ and $200 \mathrm{~mA}$ and utilising slice thickness of 3-8 mm, a matrix of $512 \times 512$ and pixel size $0.585-0.859 \mathrm{~mm}$. No intravenous or oral contrast was used.

A computed tomography (CT) range of -120 to -40 Hounsfield units (HUs) was used to encompass all fat. The total crosssectional area was calculated at each level so that intraabdominal, subcutaneous and total fat could be calculated using Adode Photoshop Elements 5 (Adobe Systems Inc., San Jose, CA, USA). In our laboratory, intra-scorer and inter-scorer (for two scorers) correlation co-efficient for CT fat assessment using Adobe is 0.98 and 0.97, respectively.

Subcutaneous adipose tissue (SAT) was defined as fat between the skin surface and the outer margin of the back and abdominal wall musculature, while intra-abdominal fat was defined as fat within the cavity formed by the back and abdominal wall musculature. We chose Adobe Photoshop over other software for abdominal fat quantification because it allows manual selection of subcutaneous/visceral fat. This is possible with the use of the "magic wand" with which we outline regions of similar signal intensity. An advantage of this method is that it allows corrections to be done by the operator, for example when the abdominal wall muscles are thin, unlike other commercially available automated packages (see online supplementary material).

CT slices at the L1 mid-lumbar level were used to assess liver steatosis by measuring the absolute number of liver attenuation values, given in HUs. According to this method, the lower the number of HUs, the lower the tissue density and therefore the greater the fat content [14].

Assessment was carried out by a single investigator who was blinded to subjects' status (i.e. control or apnoeic) or treatment phase.

Patients were asked to keep their daily routine programme, calorie/fat intake and level of physical activity steady during the study. Due to the study design, dietary habits and caloric intake/energy expenditure were not assessed on a daily basis, since this was not feasible. However, we assessed exercise habits/metabolic equivalents throughout the study phases using a standardised questionnaire [15].

\section{Blood samples}

Blood samples were drawn the morning after the first night in the sleep laboratory, at baseline and at both CPAP and sham-CPAP phases for sleep apnoea, following $12 \mathrm{~h}$ of overnight fasting. Fasting serum total cholesterol and triglyceride levels were 
assessed. Hypertriglyceridaemia was defined as serum triglyceride levels $\geqslant 150 \mathrm{mg} \cdot \mathrm{dL}^{-1}$ or the use of lipid-lowering medication.

CPAP and sham-CPAP use

All patients with OSA underwent consecutively CPAP and sham-CPAP treatment in a random order. The design of the study also included an intervening 1-week washout period between the two treatment phases. The optimal nasal CPAP pressure was determined during a full night polysomnographic study as the pressure necessary to abolish all respiratory events and snoring, secondary arousals and episodes of arterial oxygen saturation desaturation during rapid-eye movement sleep and in the supine position. During the sham-CPAP phase, patients were given an identical machine (S8 Escape, ResMed, USA), but the pressure delivered was set at the level of $1 \mathrm{cmH}_{2} \mathrm{O}$ when used with a modified mask. The mask (Ultra Mirage II nasal mask) was modified by adding an airflow restrictor and by increasing the amount of exhalation ports. CPAP usage was monitored on a daily basis by calculating the time the patient was breathing through the machine and not just the time the machine was on. In addition, a respiratory therapist visited the home of each patient regularly to provide continuous reinforcement for CPAP compliance, as well as provide us with information regarding CPAP usage (number of hours used daily, pressure settings and mask leakage). The respiratory therapist also continued to visit patients during the sham-CPAP phase. Patients, investigators and respiratory therapists were all blinded to treatment phase.

\section{Statistical analysis}

For comparisons of the groups' baseline characteristics, the independent-samples t-test was used. Because apnoeic females were significantly heavier than controls $(p<0.05)$, we employed the analysis of covariance (ANCOVA) to adjust for this difference where appropriate. Moreover, in order to deal with the issue of the significant BMI difference in our sample of apnoeic and control females, we managed to create two subgroups of apnoeic and control females, matched for BMI, and compared them in terms of abdominal fat distribution using ANOVA. As the majority of apnoeic females were slightly obese, we chose subjects with a BMI $\geqslant 28 \mathrm{~kg} \cdot \mathrm{m}^{-2}$, which resulted in a total sample of 31 subjects (13 controls and 18 apnoeic). We also used ANCOVA to compare the two groups after controlling for the effect of age, as well as age and BMI simultaneously. For comparisons between apnoeic groups' characteristics at baseline and after sham-CPAP and CPAP periods, ANOVA for repeated measurement analysis was used. Age, BMI and height data are expressed as mean $\pm \mathrm{SD}$ and the rest as mean $\pm \mathrm{SE}$.

For fat data analysis, two variables were created, total visceral adipose tissue (VAT) and total SAT areas (after summing up CT derived fat areas at every level), as indicators of total visceral/subcutaneous fat amounts. Total fat was the sum of total VAT plus total SAT.

Sex-specific linear regression analyses were developed to explore the relationship between general obesity (BMI) and abdominal fat depot variables (visceral/subcutaneous) with apnoea (AHI). The sex-specific models were carried out for each variable, i.e. BMI, total SAT, total VAT and total fat $(\mathrm{VAT}+\mathrm{SAT})$, separately, with age as the only covariate to be adjusted for in these models. To make the associations between apnoea and each of the obesity measures comparable within sex, we expressed one point increase in $\mathrm{AHI}$ as sex-specific one standard deviation increase of the adiposity variables.

Although our controls and apnoeic females were not matched in terms of BMI, we did not insert BMI in the models due to its strong association with the other adiposity indices, especially subcutaneous and total abdominal fat. Finally, in our sample of matched controls and apnoeic females, we repeated similar analysis in order to further explore these associations.

Sleep variables were calculated based on the mean values from nights 2 and 3. A p-value $<0.05$ was used to determine statistical significance. All analyses were conducted using SPSS 17.0 (SPSS Inc., Chicago, IL, USA).

\section{RESULTS}

\section{Demographic, sleep and respiratory data}

Participants' demographic and sleep characteristics are presented in table 1 .

\section{Males}

The two groups were similar in anthropometric variables, except a borderline significance for waist-hip ratio (WHR). Sleep apnoeic patients slept worse than controls. They demonstrated a significantly higher percentage of stage 1 nonrapid eye movement (NREM) sleep, wake time after sleep onset (WASO), total wake time (TWT) and a significantly lower percentage of total sleep time (TST) and stage 2 NREM sleep.

\section{Females}

No significant differences were observed in neck and waist circumference, as well as WHR after controlling for BMI. Four female controls and six apnoeic females had surgically induced menopause $(p=0.36)$. In terms of sleep, females with OSA demonstrated a significantly higher WASO, TWT and a lower percentage of TST \%. In both sexes, the 2-month CPAP treatment resulted in a significant improvement of sleep and respiratory variables, while sham-CPAP had no significant effect.

The average nightly use of CPAP was $6.01 \pm 1.19 \mathrm{~h}$ for males and $6.00 \pm 1.32 \mathrm{~h}$ for females, while 20 of the 22 apnoeic males and 18 of the 20 apnoeic females met the criteria of regular users (three subjects), and all but four patients (one male and three females) used the device for at least $4 \mathrm{~h}$ each night. Similarly, the average nightly use of sham-CPAP for both males and females was $5.18 \pm 1.42 \mathrm{~h}$ and $5.27 \pm 1.51 \mathrm{~h}$, respectively.

\section{Abdominal adipose tissue distribution and blood lipid levels} Males

Sleep apnoea patients had significantly higher amounts of intra-abdominal visceral fat at all five levels (table 2). Also, total VAT area was significantly higher in OSA patients than controls. In contrast, no significant difference was observed between the two groups at any level or in total SAT, as well as in total fat (table 2). In addition, within the control group, visceral fat amount was lower than subcutaneous fat (VAT/ SAT ratio $25^{\text {th }}$ percentile $0.46,75$ th percentile 0.88 ), while apnoeic patients presented the reverse abdominal fat deposition phenotype (VAT/SAT ratio $>1$, 25th percentile 0.88 and 75th percentile 1.20). Triglyceride blood levels and prevalence 
TABLE 1 Demographic, sleep and respiratory data in sleep apnoeic versus control subjects at baseline

\begin{tabular}{|c|c|c|c|c|c|c|}
\hline & \multicolumn{3}{|c|}{ Males } & \multicolumn{3}{|c|}{ Females } \\
\hline & Controls & Apnoeic & $\mathrm{p}$-value & Controls & Apnoeic & p-value \\
\hline Subjects n & 19 & 22 & & 20 & 20 & \\
\hline Age yrs & $52.38 \pm 6.23$ & $54.25 \pm 6.56$ & 0.36 & $54.59 \pm 5.98$ & $57.64 \pm 5.81$ & 0.12 \\
\hline BMI $\mathbf{k g} \cdot \mathrm{m}^{-2}$ & $26.60 \pm 2.65$ & $27.22 \pm 2.54$ & 0.47 & $27.85 \pm 4.24$ & $30.57 \pm 3.06$ & 0.03 \\
\hline Neck cm & $38.63 \pm 0.57$ & $39.51 \pm 0.44$ & 0.22 & $34.92 \pm 0.47$ & $35.77 \pm 0.48$ & 0.23 \\
\hline WHR & $0.95 \pm 0.05$ & $0.97 \pm 0.04$ & 0.06 & $0.86 \pm 0.13$ & $0.88 \pm 0.13$ & 0.26 \\
\hline Sleep latency min & $17.28 \pm 2.83$ & $14.24 \pm 1.58$ & 0.36 & $14.87 \pm 2.32$ & $21.49 \pm 2.32$ & 0.06 \\
\hline WASO $\min$ & $66.75 \pm 8.23$ & $112.97 \pm 10.28$ & $<0.01$ & $54.71 \pm 10.93$ & $91.31 \pm 10.93$ & 0.03 \\
\hline TWT min & $84.02 \pm 8.81$ & $127.20 \pm 10.63$ & $<0.01$ & $69.58 \pm 11.46$ & $112.80 \pm 11.46$ & 0.01 \\
\hline Sleep time TST \% & $82.56 \pm 1.82$ & $73.59 \pm 2.19$ & $<0.01$ & $85.28 \pm 8.19$ & $76.83 \pm 11.23$ & 0.01 \\
\hline REM \% & $15.05 \pm 1.10$ & $14.47 \pm 1.61$ & 0.78 & $12.47 \pm 6.42$ & $12.95 \pm 6.48$ & 0.82 \\
\hline REM latency min & $105.12 \pm 13.26$ & $109.28 \pm 14.63$ & 0.84 & $125.46 \pm 16.69$ & $141.44 \pm 16.69$ & 0.52 \\
\hline AHI & $3.03 \pm 0.44$ & $42.12 \pm 4.87$ & $<0.01$ & $1.6 \pm 0.33$ & $32.09 \pm 3.95$ & $<0.01$ \\
\hline Minimum $\mathrm{Sa}, \mathrm{O}_{2}$ & $89.00 \pm 1.12$ & $81.18 \pm 1.81$ & $<0.01$ & $91.2 \pm 0.82$ & $83.05 \pm 0.89$ & $<0.01$ \\
\hline
\end{tabular}

Data are presented as mean \pm SE, except for age, BMI and height (mean \pm SD), unless otherwise stated. Females' sleep data, waist-hip ratio (WHR), and waist and neck circumference data are reported as mean \pm SE after controlling for body mass index (BMI). WASO: wake time after sleep onset; TWT: total wake time; TST: total sleep time; N1: stage 1 nonrapid eye movement (NREM) sleep; N2: stage 2 NREM sleep; SWS: slow wave sleep; REM: rapid eye movement; AHI: apnoea/hyponoea index; Sa, ${ }_{2}$ : arterial oxygen saturation

of hypertriglyceridaemia were significantly higher in the OSA group (table 2).

\section{Females}

Visceral fat area in each separate CT slice and total VAT area were similar between controls and apnoeic subjects. In contrast, subcutaneous fat areas at L2 and L3 levels, as well as total SAT area, were significantly greater in the OSA group and there was a marginally significant trend for L1. Total fat was also significantly higher (table 2). Both apnoeic and control females were similar in terms of their sex-specific abdominal fat distribution phenotype [16, 17], with higher subcutaneous than visceral abdominal fat (controls: VAT/SAT ratio 0.39 , 25 th percentile 0.27 and 75 th percentile 0.45 ; apnoeic subjects: VAT/SAT ratio 0.38 , 25th percentile 0.27 and 75 th percentile 0.50). Apnoeic females presented a trend for a higher prevalence of hypertriglyceridaemia and higher triglyceride levels (table 2).

In the matched groups, significant differences were observed only in subcutaneous fat and total fat (table 3). The distribution of VAT/SAT ratios was also similar between the two groups.

In both sexes, liver fat content was significantly elevated in apnoeic subjects versus controls (table 2), but it was no different across sexes of the same apnoea status.

We also compared fasting blood lipid levels across sexes of the same apnoea status. No significant difference was observed in blood lipid levels between control males and females, although males had increased visceral fat. When we compared males and females with OSA in terms of fasting cholesterol or triglyceride levels, no significant difference was observed using either t-test or ANCOVA to adjust for BMI (data not shown). Sex-specific correlation analysis showed that, in both sexes, increased triglyceride levels were associated with increasing visceral fat amount (females: $r=0.51, p<0.01$; males: $\mathrm{r}=0.35, \mathrm{p}=0.03$ ) and liver fat amount (females: $\mathrm{r}=-0.31$, $\mathrm{p}=0.05$; males: $\mathrm{r}=-0.28, \mathrm{p}=0.07$ ).

Finally, no significant difference in height existed between controls and apnoeic subjects (table 1), so significant differences in fat areas could not be explained by possible height variations.

\section{Associations between apnoea and adipose tissue compartments}

Males

Within control and apnoeic males, visceral fat amount was the only variable significantly associated with apnoea (table 4). As apnoeic males and their controls were matched in general obesity (BMI), we did not anticipate seeing significant associations between general obesity and apnoea in males (see online supplementary material).

\section{Females}

All adiposity indices were significantly associated with apnoea in our sample. This may largely reflect our failure to match control and apnoeic females for BMI; this may also have limited our ability to identify visceral fat as the important variable, which we were able to do in our male sample. In this initial sample, after controlling for age, all types of abdominal 
TABLE 2 Computed tomography derived abdominal fat areas and blood lipid data in sleep apnoea patients versus controls at baseline stratified by sex

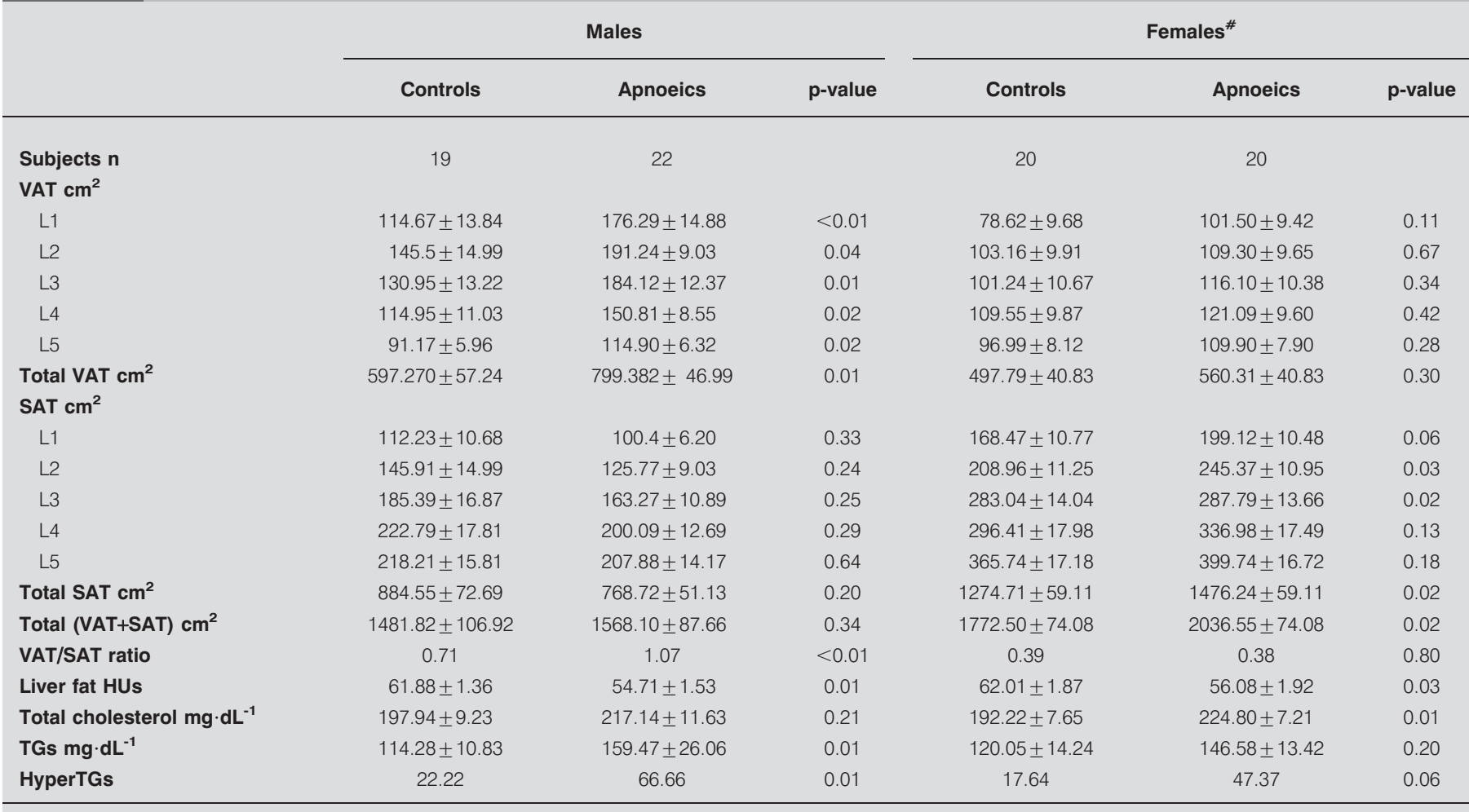

Data are expressed as mean \pm SE or $\%$, unless otherwise stated. VAT: visceral adipose tissue; SAT: subcutaneous adipose tissue. HUs: Hounsfield units; TGs: triglycerides; HyperTGs: serum triglyceride levels $\geqslant 150 \mathrm{mg} \cdot \mathrm{dL}^{-1}$ or use of lipid-lowering medication. ${ }^{\#}$ : controlled for body mass index.

fat were associated with apnoea. Similar results were found for our matched for BMI subgroup regarding abdominal fat depots (table 4).

\section{CPAP effects on sleep and respiratory variables}

The average nightly use of CPAP was $6.01 \pm 1.19 \mathrm{~h}$ for males and $6.00 \pm 1.32 \mathrm{~h}$ for females, while 20 out of the 22 apnoeic males and 18 out of the 20 apnoeic females met the criteria of regular users (three patients), and all but four patients (one male and three females) used the device for at least $4 \mathrm{~h}$ each night. Similarly, the average nightly use of sham-CPAP for both males and females was $5.18 \pm 1.42 \mathrm{~h}$ and $5.27 \pm 1.51 \mathrm{~h}$, respectively.

In both sexes, the 2-month CPAP treatment resulted in a significant improvement in sleep and respiratory variables, while sham-CPAP had no significant effect (tables 5 and 6).

TABLE 3 Characteristics of the two subgroups of apnoeic and control females with similar body mass index (BMI) range

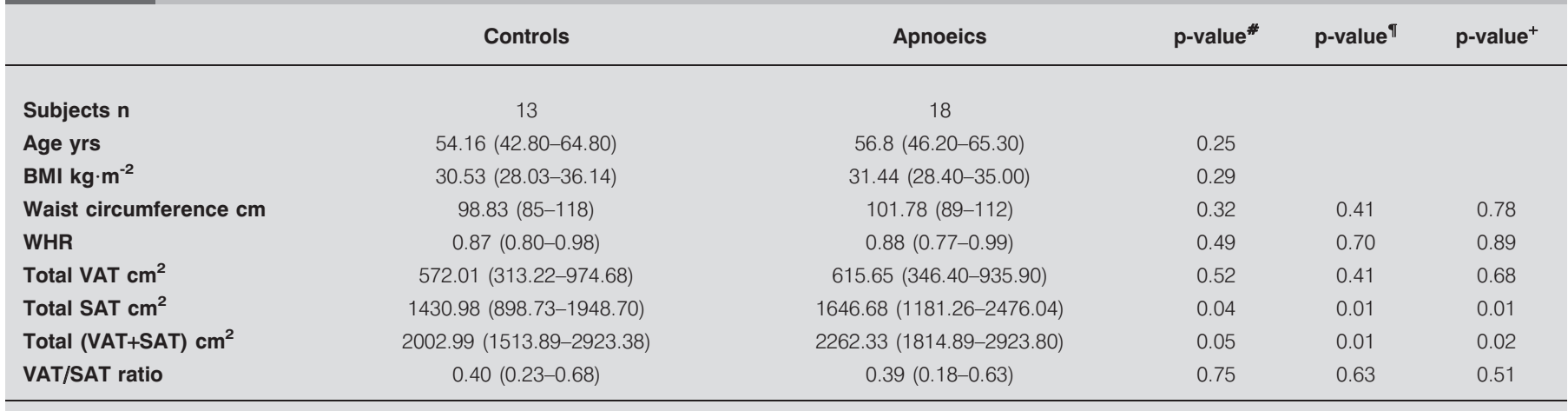

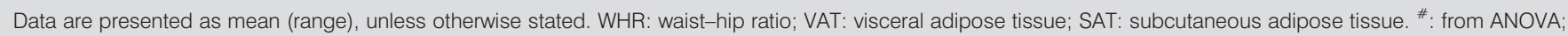
$\because$ : from ANCOVA after controlling for age; ${ }^{+}$: from ANCOVA after controlling for age and BMI. 


\begin{tabular}{|c|c|c|c|c|c|c|c|c|c|}
\hline & \multicolumn{3}{|c|}{ Males } & \multicolumn{3}{|c|}{ Females } & \multicolumn{3}{|c|}{ Females (matched group) } \\
\hline & $\beta$ & B & $p$-value & $\beta$ & B & p-value & $\beta$ & B & p-value \\
\hline Total visceral fat & 0.46 & 11.37 & $<0.01$ & 0.46 & 9.12 & $<0.01$ & 0.34 & 7.21 & 0.05 \\
\hline BMI & 0.17 & 4.55 & 0.26 & 0.43 & 8.50 & $<0.01$ & 0.33 & 6.82 & 0.07 \\
\hline
\end{tabular}

\section{CPAP effects on adiposity}

The physical activity habits of participants remained similar throughout treatment phases (data not shown). Participants' BMI did not change throughout the study. No significant change in adipose tissue distribution or amount was observed after either the CPAP or sham-CPAP periods in both sexes (tables 7 and 8). In order to explore the potential role of CPAP compliance, we performed sex-specific multiple regression analyses, where the difference in amount of fat from baseline to post-CPAP ( $\triangle \mathrm{VAT}$ for VAT and $\triangle \mathrm{SAT}$ for SAT) was the dependent variable, the hours of nightly CPAP use the predictor and the amount of fat at baseline the covariate. CPAP usage had no significant effect on either fat deposits (female $\Delta$ VAT: $\beta=0.08, p=0.75$; female $\triangle \mathrm{SAT}: \beta=-0.18$, $p=0.49$; male $\Delta$ VAT: $\beta=-0.32, p=0.18$; male $\Delta$ SAT: $\beta=0.36$, $\mathrm{p}=0.17)$. No significant change in liver fat amount was observed after the CPAP or sham-CPAP periods (tables 7 and 8). Moreover, no significant correlation existed between hours of nightly CPAP use and liver fat amount of difference

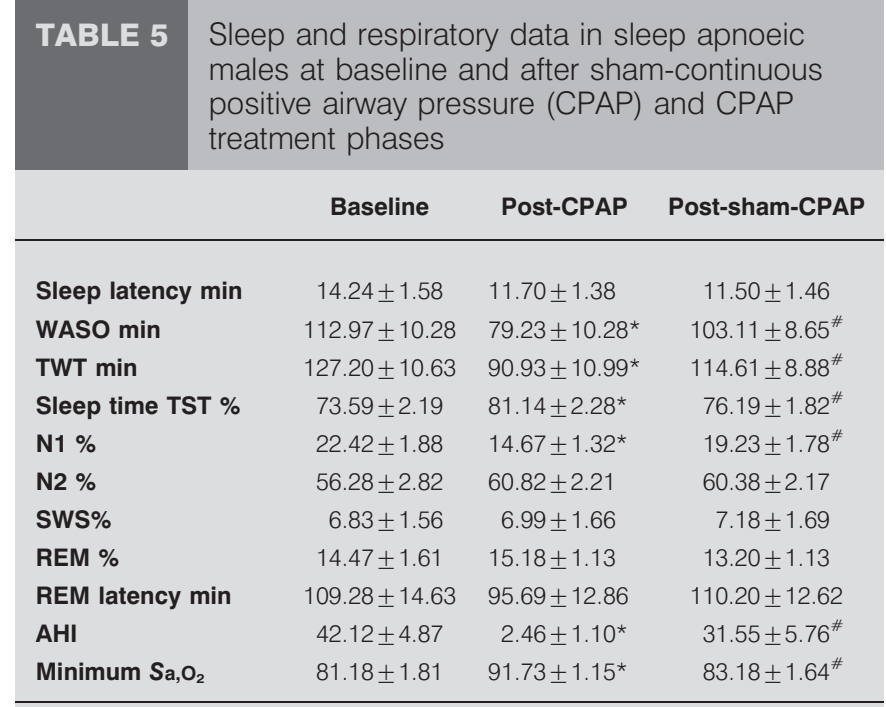

Data are presented as mean \pm SE. WASO: wake time after sleep onset; TWT: total wake time; TST: total sleep time; N1: stage 1 nonrapid eye movement (NREM) sleep; N2: stage 2 NREM sleep; SWS: slow wave sleep; REM: rapid eye movement; $\mathrm{AHI}$ : apnoea/hypopnoea index; $\mathrm{Sa}_{1} \mathrm{O}_{2}$ : arterial oxygen stauration. *: $p<0.05$ between baseline and post-CPAP treatment; ${ }^{*}: p<0.05$ between CPAP and sham-CPAP. (where the difference is the liver fat at baseline minus liver fat post-CPAP) between baseline post-CPAP $(r=-0.29, \mathrm{p}=0.24)$.

\section{DISCUSSION}

The primary findings of this study are that in overweight males, similarly to obese, visceral adiposity is the primary type of fat associated with apnoea, while in females it is global adiposity.

In apnoeic males, intra-abdominal visceral fat amounts were significantly higher than in controls, while subcutaneous abdominal fat amount was not different. Within the control group, visceral fat amount was lower than subcutaneous (VAT/SAT $<1$ ), whereas apnoeic males presented the reverse abdominal fat deposition phenotype (VAT/SAT $>1$ ), given the same total abdominal fat amount. Furthermore, only visceral fat amount was associated with apnoea severity. These findings are in accordance with and expand our previous findings in obese OSA male patients [3, 4].

TABLE 6 Sleep and respiratory data in sleep apnoeic females at baseline and after continuous positive airway pressure (CPAP) and CPAP treatment phases

\begin{tabular}{lccc} 
& Baseline & Post-CPAP & Post-sham-CPAP \\
\hline Sleep latency min & $21.15 \pm 2.55$ & $16.95 \pm 2.96$ & $18.34 \pm 3.71$ \\
WASO min & $90.45 \pm 11.98$ & $61.08 \pm 8.82^{\star}$ & $79.82 \pm 14.16$ \\
TWT min & $111.61 \pm 12.45$ & $78.03 \pm 9.76^{\star}$ & $98.16 \pm 14.85$ \\
Sleep time TST \% & $76.83 \pm 2.57$ & $83.62 \pm 2.05^{\star}$ & $79.62 \pm 3.08$ \\
N1 \% & $13.96 \pm 1.25$ & $10.10 \pm 0.87^{\star}$ & $15.09 \pm 1.76^{\#}$ \\
N2 \% & $54.74 \pm 2.40$ & $57.52 \pm 2.50$ & $57.23 \pm 2.61$ \\
SWS \% & $18.63 \pm 2.13$ & $17.59 \pm 2.42$ & $16.81 \pm 2.19$ \\
REM \% & $12.95 \pm 6.48$ & $12.92 \pm 5.85$ & $10.87 \pm 5.25$ \\
REM latency min & $139.57 \pm 15.75$ & $141.04 \pm 18.11$ & $167.01 \pm 15.82$ \\
AHI & $32.09 \pm 3.95$ & $2.15 \pm 0.62^{\star}$ & $31.82 \pm 6.07^{\#}$ \\
Minimum Sa, $\mathbf{O}_{2}$ & $83.05 \pm 0.89$ & $92.05 \pm 0.55^{\star}$ & $83.05 \pm 1.13^{\#}$ \\
\hline
\end{tabular}

Data are presented as mean \pm SE. WASO: wake time after sleep onset; TWT: total wake time; TST: total sleep time; N1: stage 1 nonrapid eye movement (NREM) sleep; N2: stage 2 NREM sleep; SWS: slow wave sleep; REM: rapid eye movement; $\mathrm{AHI}$ : apnoea/hypopnoea index; $\mathrm{Sa}_{\mathrm{O}_{2}}$ : arterial oxygen stauration. *: $p<0.05$ between baseline and post-CPAP treatment; *: $p<0.05$ between CPAP and sham-CPAP. 


\begin{tabular}{|c|c|c|c|c|c|c|c|}
\hline \multirow[t]{2}{*}{ TABLE 7} & \multicolumn{7}{|c|}{$\begin{array}{l}\text { Abdominal fat amount at baseline and after continuous positive airway pressure (CPAP) and sham-CPAP treatment } \\
\text { phases in apnoeic males }\end{array}$} \\
\hline & & Baseline & CPAP & Sham-CPAP & p-value ${ }^{*}$ & p-value & p-value ${ }^{+}$ \\
\hline \multicolumn{2}{|c|}{$B M I \mathbf{k g} \cdot \mathbf{m}^{-2}$} & $27.22 \pm 0.55$ & $27.57 \pm 0.52$ & $27.39 \pm 0.54$ & 0.13 & 1.00 & 0.67 \\
\hline \multicolumn{2}{|c|}{ Total VAT $\mathrm{cm}^{2}$} & $799.38 \pm 46.99$ & $812.79 \pm 49.43$ & $781.80 \pm 45.97$ & 1.00 & 0.90 & 0.25 \\
\hline \multicolumn{2}{|c|}{ Total SAT $\mathrm{cm}^{2}$} & $768.72 \pm 51.13$ & $794.48 \pm 51.67$ & $766.95 \pm 53.47$ & 0.22 & 1.00 & 0.23 \\
\hline \multicolumn{2}{|c|}{ Liver fat HUs } & $54.71 \pm 1.53$ & $55.43 \pm 1.51$ & $55.00 \pm 1.44$ & 1.00 & 1.00 & 1.00 \\
\hline
\end{tabular}

Data are presented as mean \pm SE, unless otherwise stated. BMI: body mass index; VAT: visceral adipose tissue; SAT: subcutaneous adipose tissue; HUs: Hounsfield units. ${ }^{*}$ : baseline versus CPAP; $"$ : baseline versus sham-CPAP; ${ }^{+}$: CPAP versus sham-CPAP.

In females, after adjustment for BMI, the only significant difference observed was in subcutaneous and total abdominal fat. Apnoea was associated with both types of fat, as well as total abdominal fat, suggesting that the most important predictor was general adiposity. This may be reflected by the fact that females with OSA, in both clinical and epidemiological samples, are heavier than apnoeic males [10], suggesting that in contrast to males, a larger degree of adiposity is necessary for females to develop OSA. We expected that in post-menopausal females with OSA, similarly to apnoeic males, visceral fat would be the prevalent abdominal fat type, i.e. they would present a VAT/SAT ratio distribution similar to our apnoeic males, as OSA and excessive daytime sleepiness are also frequent in obese and nonobese pre-menopausal females with polycystic ovary syndrome, a condition associated with visceral adiposity and insulin resistance $[18,19]$. This could be explained by the fact that although, in general population samples, the absolute visceral fat amount increases post-menopausally [16], the relative abdominal adipose tissue distribution pattern (i.e. $\mathrm{VAT} / \mathrm{SAT}$ ratio) remains relatively constant [16, 17], only changing to a more "android" type (i.e. higher visceral fat accumulation) in overweight-obese females and only at $>60 \mathrm{yrs}$ [17]. In our study, the average age of females with apnoea was $\sim 57$ yrs, which may explain the fact that visceral obesity was not detected. It is of interest that, in epidemiological studies, the prevalence of apnoea and metabolic syndrome in females peaks after the age of $60 \mathrm{yrs}$ [2].

It has been proposed that increased visceral fat amounts represent a site of shunt of excess free fatty acids due to impaired storage in subcutaneous adipocytes [20]. During this state, larger adipocytes may downregulate lipogenic genes in an attempt to limit expansion of the triglyceride stores and prevent a metabolically detrimental phenotype [21]. The worst metabolic condition of this state is represented by a higher amount of visceral than subcutaneous abdominal fat [21], which depicts a reduced capacity of even small adipocytes to store fat, leading to a greater increase in blood lipid levels, a phenotype seen in our OSA male patients. Intra-hepatic fat is also considered to be an ectopic site of lipid shunt and, along with visceral fat, has been associated with lipidaemia [22]. In the current study, we assessed fasting lipid levels; however, post-prandial hypertriglyceridaemia in both cross-sectional and prospective studies is considered to be a better predictor of increased cardiovascular morbidity and mortality [23, 24]. The association of visceral fat with lipidaemia is thought to account for the difference in blood lipid levels observed between sexes. However, we did not find such a difference between males and females of the same apnoea status, despite the difference in total visceral fat amount. This may be either due to the loss of the protective role of oestrogens post-menopausally, or it could reflect the limitation of our study, which did not assess postprandial lipid levels. Finally, visceral fat is less sensitive to insulin [25], and more cellular, vascular and innervated than subcutaneous fat, all factors predisposing towards a worse metabolic and pro-inflammatory profile.

We used CT to assess the repartition of abdominal adipose tissue, which in contrast to anthropometric indices [26, 27] or dual energy X-ray absorptiometry [28], can provide a quite

TABLE 8 Abdominal fat amount at baseline and after continuous positive airway pressure (CPAP) and sham-CPAP treatment phases in apnoeic females

\begin{tabular}{|c|c|c|c|c|c|c|}
\hline & Baseline & CPAP & Sham-CPAP & p-value ${ }^{*}$ & p-value ${ }^{\pi}$ & p-value ${ }^{+}$ \\
\hline $\mathrm{BMI} \mathbf{k g} \cdot \mathrm{m}^{-2}$ & $30.57 \pm 0.72$ & $31.21 \pm 0.77$ & $30.63 \pm 0.75$ & 0.40 & 1.00 & 0.17 \\
\hline Total SAT $\mathrm{cm}^{2}$ & $1612.92 \pm 85.40$ & $1582.86 \pm 107.79$ & $1583.78 \pm 105.11$ & 1.00 & 1.00 & 1.00 \\
\hline Total (VAT+SAT) $\mathrm{cm}^{2}$ & $2217.79 \pm 95.56$ & $2203.99 \pm 119.60$ & $2187.66 \pm 121.00$ & 1.00 & 1.00 & 1.00 \\
\hline Liver fat HUs & $53.63 \pm 3.65$ & $53.63 \pm 3.57$ & $53.38 \pm 3.47$ & 1.00 & 1.00 & 1.00 \\
\hline
\end{tabular}

Data are presented as mean $\pm \mathrm{SE}$, unless otherwise stated. BMI: body mass index; VAT: visceral adipose tissue; SAT: subcutaneous adipose tissue; HUs: Hounsfield units. $^{*}$ : baseline versus CPAP; ${ }^{\circ}$ : baseline versus sham-CPAP; ${ }^{+}$: CPAP versus sham-CPAP. 
accurate measure of specific abdominal fat compartments. Only one previous study has used CT, and it concluded that apnoeic females had a higher amount of visceral fat than controls [5]. However, the study participants were severely obese and the two groups differed significantly in terms of age (>10 yrs), a confounder that was not taken into account [16].

The effects of CPAP treatment on body composition are inconsistent. Two studies showed that CPAP can reduce the amount of visceral fat $[7,8]$. However, our negative findings are in agreement with two previous studies [4,9]. A possible explanation could be that, in our study, either the duration (2 months) was too short for a significant effect to be observed or that the study did not have enough power to detect such a difference. Nonetheless, MüNZER et al. [9], whose total study sample size was similar to ours, also did not find a significant difference, even after an 8-month CPAP treatment period. Consistent with our findings, two studies reported no improvement in weight in a clinical sample of males and females treated with CPAP after a 1-yr follow-up [29, 30].

Our findings have some important implications in terms of pathogenesis and treatment of OSA. It has been previously reported that anatomical abnormalities are very prevalent in nonobese patients with sleep apnoea, and may be causative to the pathogenesis of this disorder [31]. Our study suggests that, similarly to obese males, visceral adiposity is also the strongest factor associated with apnoea in overweight males. Furthermore, because obesity is a major risk factor for OSA in both sexes, weight loss is recommended to improve this condition in both obese/morbidly obese males and females [32, 33]. Finally, more sex-specific approaches should be developed. For example, whereas loss of visceral fat may be the primary therapeutic target in overweight males through, for example, exercise and medications, overall weight loss may be more beneficial for females [34-36].

The current study has some limitations. The most significant limitation is its failure to match apnoeic and control females in terms of BMI, However, the weight of our female participants lies at the lower end of the BMI of females with OSA typically evaluated in a sleep disorders clinic or those detected in epidemiological samples [2]. Selection of post-menopausal females was based on self-reports about absence of previous or current hormone therapy use and time since their last menstrual cycle. Moreover, its primarily cross-sectional nature cannot determine the causality of the studied associations, but we can solely report significant associations. Finally, another possible limitation of the current study is that dietary habits and calorific intake/energy expenditure were not assessed on a daily basis, as this was not feasible due to the study design. However, we assessed exercise habits/metabolic equivalents throughout the study phases using a standardised questionnaire. Moreover, the fact that the mean BMI of apnoeic males and females did not change significantly post-CPAP/postsham-CPAP indicates strongly that energy intake also remained unchanged.

In conclusion, in males, excess visceral adiposity is strongly associated with the presence and severity of apnoea, independently of general obesity. In females, global adiposity appears to be more strongly associated with apnoea status. This difference may suggest the need for sex-specific therapeutic strategies, such as reduction of visceral fat through exercise or pharmacological treatment in males $[35,36]$ and weight loss in females [34]. However, our results should be considered preliminary and point to the need for future studies in order to elucidate this issue in older post-menopausal females, where greater differences in abdominal fat distribution occur. Further studies are also needed to explore possible aetiological differences between sexes. Finally, although a 2-month CPAP treatment significantly improves sleep and respiratory events, it does not seem to affect significantly abdominal fat distribution, liver fat accumulation or total body adiposity and, thus, should be combined with other therapeutic interventions to protect sufficiently from the metabolic and inflammatory abnormalities that accompany OSA.

\section{SUPPORT STATEMENT}

This work was supported by NIH grant R01 HL 64415.

\section{STATEMENT OF INTEREST}

None declared.

\section{REFERENCES}

1 Young T, Palta M, Dempsey J, et al. The occurrence of sleepdisordered breathing among middle-aged adults. $N$ Engl J Med 1993; 328: 1230-1235.

2 Bixler EO, Vgontzas AN, Lin HM, et al. Prevalence of sleepdisordered breathing in women: effects of gender. Am J Respir Crit Care Med 2001; 163: 608-613.

3 Vgontzas AN, Papanicolaou DA, Bixler EO, et al. Chrousos GP sleep apnea and daytime sleepiness and fatigue: relation to visceral obesity, insulin resistance and hypercytokinemia. J Clin Endocrinol Metab 2000; 82: 1313-1316.

4 Vgontzas AN, Zoumakis E, Bixler EO, et al. Selective effects of CPAP on sleep apnoea-associated manifestations. Eur J Clin Invest 2008; 38: 585-595.

5 Shinohara E, Kihara S, Yamashita S, et al. Visceral fat accumulation as an important risk factor for obstructive sleep apnoea syndrome in obese subjects. J Intern Med 1997; 241: 11-18.

6 Tatsumi K, Saibara T. Effects of obstructive sleep apnea syndrome on hepatic steatosis and nonalcoholic steatohepatitis. Hepatol Res 2005; 33: 100-104.

7 Chin K, Shimizu K, Nakamura T, et al. Changes in intra-abdominal fat visceral fat and serum leptin levels in patients with OSAS following nasal continuous positive airway pressure therapy. Circulation 1999; 100: 706-712.

8 Trenell MI, Ward JA, Yee BJ, et al. Influence of constant positive airway pressure therapy on lipid storage, muscle metabolism and insulin action in obese patients with severe obstructive sleep apnoea syndrome. Diabetes Obes Metab 2007; 9: 679-687.

9 Münzer T, Hegglin A, Stannek T, et al. Effects of long-term continuous positive airway pressure on body composition and IGF1. Eur J Endocrinol 2010; 162: 695-704.

10 Lin CM, Davidson TM, Ancoli-Israel S. Gender differences in obstructive sleep apnea and treatment implications. Sleep Med Rev 2008; 12: 481-496.

11 Phillips BA, Collop NA, Drake C, et al. Sleep disorders and medical conditions in women. Proceedings of the Women \& Sleep Workshop, National Sleep Foundation, Washington, DC, March 56, 2007. J Womens Health (Larchmt) 2008; 17: 1191-1199.

12 Browning JD, Szczepaniak LS, Dobbins R, et al. Prevalence of hepatic steatosis in an urban population in the United States: impact of ethnicity. Hepatology 2004; 40: 1387-1395. 
13 Rechtschaffen A, Kales A. A Manual of Standardized Terminology, Techniques and Scoring System for Sleep Stages of Human Subjects. Washington DC, US Government Printing Office, NIMH Publication 204, 1968.

14 Yajima $Y$, Narui T, Ishii $M$, et al. Computed tomography in the diagnosis of fatty liver: total lipid content and computed tomography number. Tohoku J Exp Med 1982; 136: 337-342.

15 Wolf AM, Hunter DJ, Colditz GA, et al. Reproducibility and validity of a self-administered physical activity questionnaire. Int $J$ Epidemiol 1994; 23: 991-999.

16 Franklin RM, Ploutz-Snyder L, Kanaley JA. Longitudinal changes in abdominal fat distribution with menopause. Metabolism 2009; 58: $311-315$

17 Enzi G, Gasparo M, Biondetti PR, et al. Subcutaneous and visceral fat distribution according to sex, age, and overweight, evaluated by computed tomography. Am J Clin Nutr 1986; 44: 739-746.

18 Vgontzas AN, Legro RS, Bixler EO, et al. Polycystic ovary syndrome is associated with obstructive sleep apnea and daytime sleepiness: role of insulin resistance. J Clin Endocrinol Metab 2001; 86: 517-520.

19 Tasali E, Van Cauter E, Hoffman L, et al. Impact of obstructive sleep apnea on insulin resistance and glucose tolerance in women with polycystic ovary syndrome. J Clin Endocrinol Metab 2008; 93: 3878-3884.

20 Ravussin E, Smith SR. Increased fat intake, impaired fat oxidation, and failure of fat cell proliferation result in ectopic fat storage, insulin resistance, and type 2 diabetes mellitus. Ann NY Acad Sci 2002; 967: 363-378.

21 Kursawe R, Eszlinger $M$, Narayan D, et al. Cellularity and adipogenic profile of the abdominal subcutaneous adipose tissue from obese adolescents: association with insulin resistance and hepatic steatosis. Diabetes 2010; 59: 2288-2296.

22 Musso G, Gambino R, Durazzo M, et al. Adipokines in NASH: postprandial lipid metabolism as a link between adiponectin and liver disease. Hepatology 2005; 42: 1175-1183.

23 Bansal S, Buring JE, Rifai N, et al. Fasting compared with nonfasting triglycerides and risk of cardiovascular events in women. JAMA 2007; 298: 309-316.
24 Nordestgaard BG, Benn M, Schnohr P, et al. Nonfasting triglycerides and risk of myocardial infarction, ischemic heart disease, and death in men and women. JAMA 2007; 298: 299-308.

25 Saltiel AR, Kahn CR. Insulin signalling and the regulation of glucose and lipid metabolism. Nature 2001; 414: 799-806.

26 Millman RP, Carlisle CC, McGarvey ST, et al. Body fat distribution and sleep apnea severity in women. Chest 1995; 107: 362-366.

27 Martinez-Rivera C, Abad J, Fiz JA, et al. Usefulness of truncal obesity indices as predictive factors for obstructive sleep apnea syndrome. Obesity (Silver Spring) 2008; 16: 113-118.

28 Soriano-Co M, Vanhecke TE, Franklin BA, et al. Increased central adiposity in morbidly obese patients with obstructive sleep apnea. Intern Med J 2011; 41: 560-566.

29 Redenius R, Murphy C, O'Neill E, et al. Does CPAP lead to change in BMI? J Clin Sleep Med 2008; 4: 205-209.

30 Quan SF. Proceedings of the National Sleep Foundation meeting. Washington DC, National Sleep Foundation, 2006.

31 Vgontzas AN. Does obesity play a major role in the pathogenesis of sleep apnoea and its associated manifestations via inflammation, visceral adiposity, and insulin resistance? Arch Physiol Biochem 2008; 114: 211-223.

32 Johansson K, Neovius M, Lagerros YT, et al. Effect of a very low energy diet on moderate and severe obstructive sleep apnoea in obese men: a randomised controlled trial. BMJ 2009; 339: b4609.

33 Romero-Corral A, Caples SM, Lopez-Jimenez F, et al. Interactions between obesity and obstructive sleep apnea: implications for treatment. Chest 2010; 137: 711-719.

34 Foster GD, Borradaile KE, Sanders $\mathrm{MH}$, et al. Sleep AHEAD Research Group of Look AHEAD Research Group. A randomized study on the effect of weight loss on obstructive sleep apnea among obese patients with type 2 diabetes: the Sleep AHEAD study. Arch Intern Med 2009; 169: 1619-1626.

35 Phillips CL, Yee BJ, Trenell MI, et al. Changes in regional adiposity and cardio-metabolic function following a weight loss program with sibutramine in obese men with obstructive sleep apnea. J Clin Sleep Med 2009; 5: 416-421.

36 Totsikas C, Röhm J, Kantartzis K, et al. Cardiorespiratory fitness determines the reduction in blood pressure and insulin resistance during lifestyle intervention. J Hypertens 2011; 29: 1220-1227. 